\title{
A prevalência do uso de sonda nasogástrica em bebês portadores de fissura de lábio e/ou palato
}

\author{
Prevalence of the use of nasogastric tube in babies with cleft lip \\ and/or palate
}

\author{
Camila Queiroz de Moraes Silveira Di Ninno', Fernanda Cristina de Faria Vieira ${ }^{2}$, Angélica Maria Moreira Lemos ${ }^{2}$, \\ Luana Farnezi Silva ${ }^{2}$, Christiane Marize Garcia Rocha ${ }^{3}$, Rocksane de Carvalho Norton ${ }^{4}$, Cíntia Santos Silva \\ Machado 5 , Denise Brandão de Oliveira e Britto ${ }^{6}$
}

\begin{abstract}
RESUMO
Objetivo: Investigar a prevalência do uso de sonda nasogástrica em bebês com fissura labiopalatina, sua correlação com tipo de fissura, maternidade e cidade de origem, e a idade na primeira consulta. Métodos: A amostra constituiu-se de 137 bebês de ambos os gêneros, com fissura de lábio e/ou palato, sem outros comprometimentos, nascidos a termo, e que chegaram para primeira consulta em um centro especializado em fissura entre zero e 12 meses (mediana=33 dias). Realizou-se análise estatística pelo teste de coeficiente de contingência $(\mathrm{p}<0,05)$. Resultados: Da amostra total, $61 \%$ eram do gênero masculino e $39 \%$ do feminino, $51 \%$ apresentavam fissura de lábio e palato, $35 \%$ de palato e $14 \%$ de lábio. Quanto ao nascimento, $36 \%$ nasceram em maternidades particulares e $64 \%$ em públicas, $60 \%$ em Belo Horizonte, $15 \%$ em outras cidades da região metropolitana e $25 \%$ no interior do estado. O uso de sonda ocorreu em $23 \%$ dos casos. Não houve associação entre tipo de fissura ou de maternidade e o uso de sonda, mas este foi mais frequente na região metropolitana ( $\mathrm{p}=0,007)$. Conclusão: A prevalência do uso de sonda em bebês com fissura foi considerada alta, visto que nasceram a termo e não apresentavam comprometimentos associados que indicassem o uso da mesma. O uso de sonda é mais frequente em bebês nascidos em maternidades da região metropolitana de Belo Horizonte, quando comparados a outras cidades do estado de Minas Gerais.
\end{abstract}

Descritores: Fenda labial; Fissura palatina; Recém-nascido; Métodos de alimentação; Nutrição enteral

\section{INTRODUÇÃO}

Bebês portadores de fissura de lábio e/ou palato podem

Trabalho realizado no Curso de Fonoaudiologia da Pontifícia Universidade Católica de Minas Gerais - PUC-Minas - Belo Horizonte (MG), Brasil.

(1) Doutora, Professora Adjunto do Curso de Fonoaudiologia da Pontifícia Universidade Católica de Minas Gerais - PUC-Minas - Belo Horizonte (MG), Brasil.

(2) Acadêmica do Curso de Fonoaudiologia da Pontifícia Universidade Católica de Minas Gerais - PUC-Minas - Belo Horizonte (MG), Brasil.

(3) Mestre, Médica pediatra do Centro de Tratamento e Reabilitação de Fissuras Labiopalatinas e Deformidades Craniofaciais - PUC-Minas/Hospital da Baleia - Belo Horizonte (MG), Brasil.

(4) Doutora, Professora da Faculdade de Medicina da Universidade Federal de Minas Gerais - UFMG - Belo Horizonte (MG), Brasil.

(5) Mestre, Professora Assistente III do Curso de Fonoaudiologia da Pontifícia Universidade Católica de Minas Gerais - PUC-Minas - Belo Horizonte (MG), Brasil.

(6) Pós-graduanda (Doutorado) em Letras pela Pontifícia Universidade Católica de Minas Gerais - PUC-Minas - Belo Horizonte (MG), Brasil. Professora Assistente III do Curso de Fonoaudiologia da Pontifícia Universidade Católica de Minas Gerais - PUC-Minas - Belo Horizonte (MG), Brasil.

Endereço para correspondência: Camila Queiroz de Moraes Silveira Di Ninno. Av. Dom José Gaspar, 500 - Prédio 25, Coração Eucarístico, Belo Horizonte (MG), Brasil, CEP: 30535-610. E-mail: camilaninno@uol.com.br Recebido em: 13/6/2009; Aceito em: 2/5/2010 apresentar um desenvolvimento bem próximo do normal se receberem intervenção adequada desde o nascimento ${ }^{(1)}$

No entanto, isso nem sempre acontece, pois o desconhecimento de muitos profissionais da área da saúde em relação às fissuras de lábio e/ou palato impossibilita que vários bebês usufruam dos benefícios da intervenção precoce ${ }^{(2)}$.

Após o nascimento de um bebê com fissura de lábio e/ou palato é preciso traçar estratégias de tratamento e fazer a escolha da abordagem alimentar mais adequada à sua capacidade. É indiscutível o valor do aleitamento materno, uma vez que este leite possui características nutritivas e antibacterianas que auxiliam no combate a infecções comuns nos portadores de fissura. Se não for possível o aleitamento no seio, recomenda-se que o leite materno seja ordenhado e oferecido na mamadeira, ou então que seja introduzido outro tipo de leite indicado pelo pediatra $^{(3)}$.

A alimentação oral deve ser estimulada precocemente, ou seja, logo após o nascimento, sob a orientação de equipe especializada ou treinada ${ }^{(4)}$. Esses cuidados aumentam as chances de se alcançar melhores condições de saúde do bebê, possibilitando as intervenções cirúrgicas na idade ideal e evitando, com isso, futuras complicações ${ }^{(4,5)}$.

Ainda na maternidade, se possível, deve ocorrer o primeiro 
contato entre o fonoaudiólogo, o bebê com fissura e sua família ${ }^{(1)}$. É neste momento que os pais precisam, além do apoio emocional, esclarecer suas dúvidas e receber orientações sobre como proceder em relação à alimentação do bebê( $\hat{e}^{()}$.

A ausência de integridade anatômica da cavidade oral pode trazer prejuízos ao mecanismo de sucção, dificultando a alimentação do bebê com fissura ${ }^{(7-9)}$, que passa a ser um processo trabalhoso, demorado e que provoca ansiedade nos pais pelo fato do bebê ingerir, muitas vezes, um volume de líquido insuficiente ${ }^{(10)}$. A preocupação excessiva dos pais, por sua vez, é também apontada como um fator que pode aumentar ainda mais a dificuldade alimentar do bebê $\hat{e}^{(11)}$.

As dificuldades alimentares não são comuns a todos os bebês portadores de fissura de lábio e/ou palato e podem estar relacionadas com as estruturas acometidas pela fissura( ${ }^{(4,9,12,13)}$. Sabe-se que bebês com fissura do tipo pré-forame incisivo conseguem, com maior frequência, ser alimentados no seio materno $^{(1,4,10-14)}$ e que bebês com fissura do tipo pós-forame incisivo e transforame incisivo, apresentam maior dificuldade, principalmente devido à fraca pressão intra-oral para a sucção ${ }^{(1,9-13)}$. Alguns estudos apontam que bebês com fissura de palato podem apresentar maior dificuldade alimentar do que os com fissura de lábio e palato ${ }^{(11,12)}$.

Em bebês com fissura de palato, mas sem síndromes associadas, a dificuldade alimentar presente ao nascimento costuma ser superada nos primeiros meses de vida. Como nem toda síndrome é facilmente diagnosticada ao nascimento, a persistência de dificuldades alimentares após os três meses de vida pode ser um sinal da presença de outros comprometimentos associados ${ }^{(12)}$.

Várias são as indicações de instrumentos que visam auxiliar os bebês com fissura labiopalatina durante o processo alimentar, mas nenhum deles tem aceitação universal. Dentre estes instrumentos, podemos encontrar o uso de mamadeira, copinho, colher, conta-gotas, seringa e sonda ${ }^{(1,4,7,11,15-18)}$. O uso de sondas, conta-gotas e seringas, no entanto, é contra-indicado por muitos profissionais, por julgarem não ser necessário o uso de instrumentos especiais para a alimentação da grande maioria de bebês com fissura ${ }^{(15-17)}$.

Na prática clínica, não é raro encontrarmos bebês portadores de fissura labiopalatina, não sindrômicos e nascidos a termo, que por algum motivo foram submetidos à nutrição enteral, em especial por meio de sondas nasogástricas (SNG). Vários estudos da literatura que investigaram a alimentação de bebês com fissura de lábio e/ou palato também encontraram casos que fizeram uso de SNG ao nascimento ${ }^{(4,11,12,18)}$.

Entende-se por nutrição enteral um conjunto de procedimentos terapêuticos que são empregados para a manutenção ou recuperação do estado nutricional, na qual a via digestiva, fisiológica, é utilizada para receber dietas especiais que são administradas por SNG, orogástricas ou transpilórica ${ }^{(19)}$. A nutrição enteral é considerada uma ferramenta nutricional poderosa, mas que contém complicações intrínsecas podendo limitar o seu uso ${ }^{(18)}$.

A SNG apresenta, como vantagem, uma fixação mais fácil e duradoura, mas pode provocar aumento na resistência das vias aéreas, levando inclusive à ocorrência de apneias. Além disso, podem retardar a transição para a alimentação por via oral ${ }^{(20)}$, interferir nos mecanismos normais de respiração no período neonatal, que dependem do reflexo nasoalveolar, devido à interposição da sonda na narina e comprometer a sensibilidade da cavidade oral, o que atrasa o início do desenvolvimento da habilidade de sucção ${ }^{(21)}$.

Um bebê, ao utilizar a sonda para se alimentar, tem prejudicados os seus mecanismos próprios para realizar os movimentos de sucção e deglutição. O desenvolvimento da coordenação sucção-deglutição-respiração pode ser comprometido pelo uso prolongado de SNG, podendo levar também a dificuldades posteriores durante o estabelecimento da alimentação por via oral.

A nutrição enteral por meio de sondas é mais indicada para recém-nascidos com idade gestacional abaixo de 34 semanas ou com peso inferior a $1500 \mathrm{~g}$; doenças neurológicas; doenças cardiorespiratórias; malformações maxilo-faciais; incapacidade de sucção e/ou deglutição; atresia de esôfago; fístula traqueoesofágica; entre outras ${ }^{(22)}$. Embora na literatura as fissuras que envolvem o palato estejam entre os problemas que podem levar ao uso de sonda ${ }^{(22)}$, a indicação da SNG para alimentação deve ser restrita aos casos em que não se obtém sucesso com as tentativas de alimentação por via oral ${ }^{(4)}$. Tal indicação acaba ocorrendo em poucos casos, e não tem relação com o defeito congênito em si, mas sim com outras anomalias sobrepostas $^{(5,18)}$.

Bebês com fissura de lábio e/ou palato, nascidos a termo e sem qualquer outra alteração associada, podem e devem ser alimentados da forma mais próxima possível do normal, desde as primeiras horas de vida, não necessitando, portanto, do uso de $\mathrm{SNG}^{(1)}$. A alimentação por SNG nos bebês portadores de fissura de lábio e/ou palato pode se tornar uma experiência desagradável e desconfortável, tanto para o bebê, como para a mãe.

Para que o bebê obtenha a coordenação entre respiração, sucção e deglutição, pode ser utilizada, como estímulo, a sucção não-nutritiva por meio do uso de chupeta e a sucção nutritiva, sendo que com esta última o bebê conseguirá uma adequada coordenação entre as funções. Pesquisa realizada com bebês demonstrou que quando a chupeta é oferecida durante a alimentação por SNG, a alimentação por via oral começa mais cedo do que quando não há sucção não-nutritiva associada, os bebês engordam mais e recebem alta hospitalar mais cedo ${ }^{(23)}$.

Uma vez que é comum encontrarmos bebês que utilizaram SNG em virtude da presença de fissura de lábio e/ou palato, e que estes nem sempre teriam a indicação para tal intervenção, faz-se necessário desmistificar a necessidade do uso da SNG nos portadores de fissura e orientar pais e profissionais da área da saúde quanto à compreensão de que o recém-nascido a termo com fissura de lábio e/ou palato isolada não necessita do uso de SNG.

O presente estudo teve por objetivo investigar a prevalência do uso de SNG em bebês portadores de fissura de lábio e/ou palato, nascidos a termo, correlacionando o uso da sonda com o tipo de fissura, o tipo de maternidade (particular ou pública) e a cidade de origem. Além disso, objetivou-se quantificar o tempo transcorrido entre o nascimento e a primeira consulta em um serviço especializado. 


\section{MÉTODOS}

Este estudo, aprovado pelo Comitê de Ética em Pesquisa da Pontifícia Universidade Católica de Minas Gerais, sob o número CAAE - 0114.0.213.000-07, foi realizado por meio da coleta de dados nos arquivos da Pediatria de um Centro de Tratamento e Reabilitação de Fissuras Labiopalatinas e Deformidades Craniofaciais.

A amostra deste estudo foi constituída por 137 prontuários de pacientes com fissura de lábio e/ou palato do Centro de Tratamento e todos os responsáveis pelos participantes assinaram um Termo de Consentimento Livre e Esclarecido. Como critérios de inclusão, foram selecionados prontuários de bebês de ambos os gêneros, com fissura de lábio e/ou palato, nascidos a termo e que chegaram para a primeira consulta no setor de pediatria do Centro de Tratamento com idade entre zero e 12 meses. Foram excluídos os casos que apresentavam síndromes, cardiopatias e comprometimento do sistema nervoso central, associados e, inclusive, os bebês com diagnóstico clínico de Sequência de Pierre Robin, o que poderia indicar o uso de sondas de alimentação ${ }^{(5,18,24)}$.

Foram coletados dados relativos ao gênero, tipo de fissura diagnosticada, tempo transcorrido entre o nascimento e a primeira consulta, tipo de alimentação indicada na maternidade, incluindo o uso ou não de SNG, tipo de maternidade (particular ou pública) e a cidade de origem, colhidos na primeira consulta do bebê no Centro de Tratamento.

A classificação das fissuras de lábio e/ou palato utilizada neste estudo foi a proposta por Spina et al. ${ }^{(25)}$, que divide as fissuras em quatro grupos: fissura pré-forame incisivo, fissura pós-forame incisivo, fissura transforame incisivo e fissuras raras de face.

Após a tabulação dos dados, estes foram analisados por meio do teste de coeficiente de contingência, com nível de significância de 5\% ( $<<0,05)$, a partir do software estatístico Statistical Package for the Social Sciences (SPSS) versão 9.0.

\section{RESULTADOS}

Dos 137 prontuários analisados, 83 (61\%) eram relativos a bebês do gênero masculino e 54 (39\%) do gênero feminino. A Figura 1 sintetiza os tipos de fissura apresentados.

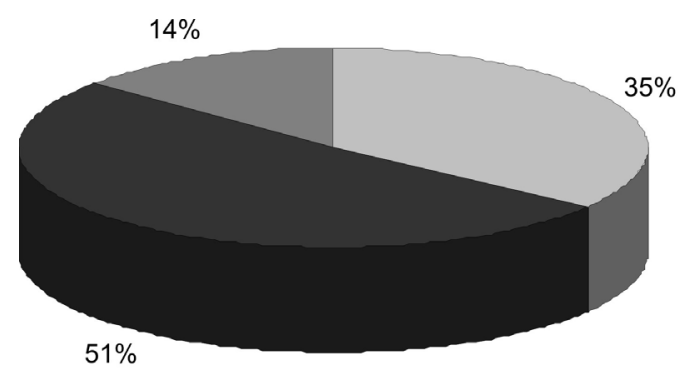

$\square$ Fissura pós-forame

- Fissura transforame Fissura pré-forame

Figura 1. Distribuição da amostra por tipo de fissura
Em relação à naturalidade, 82 (60\%) bebês nasceram em Belo Horizonte, 21 (15\%) em outras cidades da região metropolitana de Belo Horizonte e 34 (25\%) em cidades do interior do Estado de Minas Gerais. Cinquenta bebês (36\%) nasceram em maternidades particulares e 87 (64\%) em maternidades públicas. Quanto ao uso da SNG, 31 bebês (23\%) tiveram indicação, na maternidade, para o uso de sonda.

O tempo transcorrido entre o nascimento dos bebês e a primeira consulta no Centro de Tratamento teve variação média de 53 dias (DP=51dias) e mediana de 33 dias.

Para a análise dos dados, a amostra estudada foi subdividida em dois grupos: grupo 1, representado pelos bebês que não usaram SNG e grupo 2, pelos bebês que usaram a sonda (Figura 2).

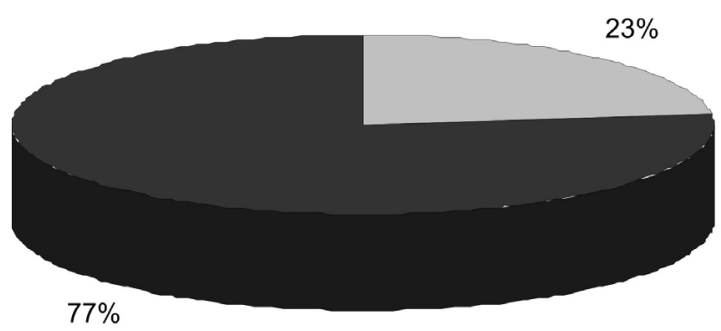

$\square$ G2 $\square$ G1

Legenda: G1 = Grupo 1: não usou sonda; G2 = Grupo 2: usou sonda

Figura 2. Distribuição da porcentagem de bebês por uso de sonda nasogástrica

Na Tabela 1, encontram-se distribuídos os dados relativos às variáveis de gênero, tipo de fissura, naturalidade e maternidade, de acordo com os grupos.

De acordo com a análise estatística realizada, não houve associação $(\mathrm{p}=0,181)$ entre o tipo de fissura e o uso de SNG (Tabela 2). Também não houve associação $(\mathrm{p}=0,983)$ entre o tipo de maternidade e o uso de SNG (Tabela 3). Em relação à naturalidade, houve evidências $(\mathrm{p}=0,007)$ de que exista associação entre o local de nascimento e o uso de SNG (Tabela 4). Quanto à idade no dia da primeira consulta ao pediatra, verificou-se diferença $(\mathrm{p}=0,001)$ entre os grupos (Tabela 5).

\section{DISCUSSÃO}

$\mathrm{Na}$ amostra analisada, o predomínio de bebês do gênero masculino e de fissura do tipo transforame incisivo está de acordo com estudos da literatura que abordam a prevalência de fissura na população ${ }^{(26,27)}$.

O fato de o Centro de Tratamento ser um dos únicos centros de referência no atendimento a pacientes com fissura do estado de Minas Gerais justifica termos, no presente estudo, uma grande porcentagem de casos nascidos no interior do Estado.

Neste trabalho, o maior número de nascimentos ocorreu em maternidades públicas, o que pode ser explicado pelo Centro de Tratamento ter todos os seus atendimentos custeados pelo Sistema Único de Saúde (SUS), o que viabiliza o acesso de 
Tabela 1. Distribuição por gênero, tipo de fissura, naturalidade e tipo de maternidade

\begin{tabular}{|c|c|c|c|c|c|}
\hline \multirow[t]{2}{*}{ Grupos } & & \multicolumn{2}{|c|}{ G1 } & \multicolumn{2}{|c|}{ G2 } \\
\hline & & $\mathrm{N}$ & $\%$ & $\mathrm{~N}$ & $\%$ \\
\hline \multirow[t]{3}{*}{ Gênero } & Masculino & 59 & 56 & 24 & 77 \\
\hline & Feminino & 47 & 44 & 7 & 23 \\
\hline & Total & 106 & 100 & 31 & 100 \\
\hline \multirow[t]{4}{*}{ Tipo de fissura } & Pré-forame & 17 & 16 & 2 & 7 \\
\hline & Transforame & 51 & 48 & 19 & 61 \\
\hline & Pós-forame & 38 & 36 & 10 & 32 \\
\hline & Total & 106 & 100 & 31 & 100 \\
\hline \multirow[t]{4}{*}{ Naturalidade } & Belo Horizonte & 58 & 55 & 24 & 78 \\
\hline & Região metropolitana & 15 & 14 & 6 & 19 \\
\hline & Interior & 33 & 31 & 1 & 3 \\
\hline & Total & 106 & 100 & 31 & 100 \\
\hline \multirow[t]{3}{*}{ Maternidade } & Particular & 39 & 37 & 11 & 35 \\
\hline & Pública & 67 & 63 & 20 & 65 \\
\hline & Total & 106 & 100 & 31 & 100 \\
\hline
\end{tabular}

Legenda: G1 = Grupo 1: não usou sonda; G2 = Grupo 2: usou sonda; N = número de indivíduos

Tabela 2. Uso de sonda nasogástrica por tipo de fissura

\begin{tabular}{lcccc}
\hline \multirow{3}{*}{ Grupos } & \multicolumn{3}{c}{ Tipo de fissura } & Total \\
\cline { 2 - 4 } & $\begin{array}{c}\text { Pré-forame } \\
\mathrm{N}(\%)\end{array}$ & $\begin{array}{c}\text { Transforame } \\
\mathrm{N}(\%)\end{array}$ & $\begin{array}{c}\text { Pós-forame } \\
\mathrm{N}(\%)\end{array}$ \\
\hline $\mathrm{G} 1$ & $17(89)$ & $51(72)$ & $38(79)$ & $106(77)$ \\
$\mathrm{G} 2$ & $2(11)$ & $19(27)$ & $10(21)$ & $31(23)$ \\
\hline Total & 19 & 70 & 48 & 137 \\
\hline
\end{tabular}

Legenda: G1 = Grupo 1: não usou sonda; G2 = Grupo 2: usou sonda

Tabela 4. Uso de sonda nasogástrica por cidade de nascimento

\begin{tabular}{lcccc}
\hline & \multicolumn{3}{c}{ Naturalidade } & \\
\cline { 2 - 4 } Grupos & $\begin{array}{c}\text { Belo } \\
\text { Horizonte } \\
\end{array}$ & $\begin{array}{c}\text { Região } \\
\text { metropolitana }\end{array}$ & $\begin{array}{c}\text { Interior } \\
\text { N }(\%)\end{array}$ & $\begin{array}{c}\text { Total } \\
\text { N }(\%)\end{array}$ \\
\hline G1 & $58(71)$ & $15(71)$ & $33(97)$ & $106(77)$ \\
G2 & $24(29)$ & $6(29)$ & $1(3)$ & $31(23)$ \\
\hline Total & 82 & 21 & 34 & 137 \\
\hline
\end{tabular}

Legenda: G1 = Grupo 1: não usou sonda; G2 = Grupo 2: usou sonda

pacientes pertencentes às classes sócio-econômicas menos favorecidas.

Embora a média de idade dos bebês, na primeira consulta no Centro, tenha sido de 53 dias, acreditamos que a mediana (33 dias) seja mais representativa da amostra, uma vez que o desvio padrão foi alto (51 dias). Assim, pode-se notar que metade dos bebês chegou para a primeira consulta com até um mês de idade. Este achado está de acordo com outro estudo também realizado na região sudeste do Brasil, o qual identificou que os bebês com fissura chegavam ao centro especializado com idades entre quatro e 60 dias de vida $^{(4)}$. Pensando na importância de um trabalho de intervenção precoce ${ }^{(1,28,29)}$, seria desejável que todos os bebês fossem encaminhados diretamente das maternidades para serviços especializados e,
Tabela 3. Uso de sonda nasogástrica por tipo de maternidade

\begin{tabular}{lccc}
\hline & \multicolumn{2}{c}{ Tipo de maternidade } & Total \\
\cline { 2 - 3 } Grupos & $\begin{array}{c}\text { Pública } \\
\mathrm{N}(\%)\end{array}$ & $\begin{array}{c}\text { Particular } \\
\mathrm{N}(\%)\end{array}$ & $\mathrm{N}(\%)$ \\
\hline $\mathrm{G} 1$ & $39(78)$ & $67(77)$ & $106(77)$ \\
G2 & $11(22)$ & $20(23)$ & $31(23)$ \\
\hline Total & 50 & 87 & 137 \\
\hline
\end{tabular}

Legenda: G1 = Grupo 1: não usou sonda; G2 = Grupo 2: usou sonda

Tabela 5. Estatística descritiva para a idade no dia da primeira consulta em dias, de acordo com o uso de sonda nasogástrica

\begin{tabular}{lccccc}
\hline Grupos & Média & DP & Mínimo & Mediana & Máximo \\
\hline G1 & 59 & 53 & 2 & 41 & 307 \\
G2 & 32 & 33 & 2 & 18 & 114 \\
\hline Total & 53 & 51 & 2 & 33 & 307 \\
\hline Valor de $p$ & $0,0001^{*}$ & - & - & - & - \\
\hline
\end{tabular}

* Valor significativo

Legenda: G1 = Grupo 1: não usou sonda; G2 = Grupo 2: usou sonda

portanto, que todos chegassem para a primeira consulta antes de um mês de idade.

Quando os bebês foram divididos em dois grupos, de acordo com o uso ou não de SNG, observou-se que os bebês que fizeram uso de SNG ao nascimento chegaram ao centro especializado mais cedo do que os demais. Isto pode ter ocorrido em função da busca precoce por orientações alimentares, fato este não investigado na presente pesquisa.

Em relação ao uso de SNG, encontramos quase um quarto dos pacientes com histórico de uso de sonda ao nascimento. $\mathrm{Na}$ literatura pesquisada, outros estudos também encontraram muitos bebês com fissura que tinham feito uso de SNG ao nascimento, em porcentagens que variavam entre e $17 \%$ e $72 \%(4,11,12,18)$. Esta grande diferença entre os achados pode ser 
justificada pelos diferentes critérios de inclusão e exclusão dos bebês adotados nos referidos estudos. Alguns incluíam todos os bebês com fissura de lábio e/ou palato e outros excluíam da amostra os bebês com outras anomalias associadas. No presente estudo, optou-se por excluir bebês com a Sequência de Pierre Robin, pois estes casos com frequência necessitam do uso de SNG, ao menos nos primeiros dias de vida ${ }^{(18,24,30)}$.

$\mathrm{Na}$ amostra pesquisada, a porcentagem de bebês que fizeram uso de SNG foi considerada alta, visto que, de acordo com a avaliação da pediatra da instituição na primeira consulta, nenhum dos bebês teria real necessidade de uso da sonda. Vale lembrar que foram excluídos bebês prematuros e com qualquer outra anomalia associada, inclusive com a Sequência de Pierre Robin. No entanto, não tivemos acesso aos prontuários das maternidades em que os bebês nasceram, o que poderia ter ajudado a esclarecer o motivo da indicação da SNG. Os autores de um estudo que encontrou $17 \%$ dos casos com uso da SNG por um período entre um e 95 dias, também consideraram o valor alto e inesperado, mas justificam o achado, em parte, por não terem excluído os casos com Sequência de Pierre Robin. Levantaram também a hipótese de que, em geral, o uso de SNG esteja aumentando nos últimos anos, embora não tenham investigado este fato ${ }^{(18)}$.

Outros autores concordam que pacientes com fissura de lábio e/ou palato apresentam condições de serem alimentados por via oral desde o nascimento e que o uso da SNG só é necessário em casos de dificuldades alimentares extremas, geralmente em bebês com outras anomalias associadas ${ }^{(1,4,10,29,30)}$. O uso da SNG só deveria ser indicado quando outros métodos alimentares não forem efetivos para garantir a nutrição e o ganho de peso do paciente ${ }^{(4,29,30)}$. Entretanto, muitas vezes seu uso acaba sendo indicado por ser a forma mais rápida de minimizar dificuldades de ganho de peso $^{(4)}$. Pesquisas realizadas no Brasil chamam a atenção para a necessidade de treinamento dos profissionais da saúde de maternidades, bem como a necessidade de encaminhar estes bebês precocemente para centros especializados ${ }^{(2,4)}$. Por outro lado, estudo desenvolvido na Alemanha mostra os benefícios de se poder contar com profissionais da saúde treinados para intervir junto a bebês com fissura e seus familiares, tanto ao nascimento, nas maternidades, como posteriormente, em casa ${ }^{(18)}$.

Apesar de não ter havido associação entre o tipo de fissura e o uso de SNG, pode-se observar uma tendência ao menor uso de SNG em bebês com fissura do tipo pré-forame incisivo. Talvez o número reduzido de bebês com este tipo de fissura na amostra estudada tenha interferido nesta análise. Acredita-se que, com uma amostra maior, a associação entre estas variáveis possa ser encontrada. Sabe-se que bebês com fissura préforame, por apresentarem integridade do palato, apresentam menores dificuldades para amamentar ${ }^{(1,4,9-14,29)}$. Na literatura há relato de uso mais frequente de SNG em pacientes com fissura labiopalatina e palatina ${ }^{(4,11,12)}$, tanto pela dificuldade em obter pressão intra-oral durante a sucção, como por estar esta última mais relacionada a casos sindrômicos e à sequência de Pierre Robin ${ }^{(30)}$.

Quanto às variáveis de uso de SNG e tipo de maternidade (particular ou pública), não foram encontradas evidências de associação entre elas, uma vez que a proporção de bebês que utilizou SNG em maternidades particulares é muito parecida com a que utilizou em maternidades públicas. Assim sendo, estratégias devem ser traçadas visando esclarecer profissionais da área da saúde a respeito da alimentação de bebês nascidos com fissura de lábio e/ou palato, tanto em maternidades particulares como em públicas.

Em relação à cidade de nascimento do paciente, no presente estudo o uso da SNG foi menor em bebês nascidos no interior do estado de Minas Gerais, quando comparados à capital e demais cidades da região metropolitana de Belo Horizonte. Estudo anterior mostrou um desconhecimento por parte dos profissionais da área de saúde de maternidades da cidade de Belo Horizonte sobre vários aspectos específicos das fissuras de lábio e/ou palato ${ }^{(2)}$, o que poderia justificar a indicação excessiva da SNG. Por desconhecer a melhor forma de alimentar um bebê com fissura, o pediatra poderia em um primeiro momento passar a sonda de alimentação, enquanto aguarda a avaliação do fonoaudiólogo quanto às condições de sucção e deglutição do bebê. No interior do estado, tal protocolo de atendimento não existiria, provavelmente pela escassez de fonoaudiólogos nas maternidades. Esta explicação é uma hipótese levantada pelos autores, uma vez que neste estudo, o motivo da indicação da SNG não foi averiguado.

Desta forma, sugere-se a continuidade deste estudo visando investigar mais profundamente a razão deste achado. Seria interessante identificar por quanto tempo a SNG foi utilizada, se há determinadas maternidades ou profissionais da área da saúde, responsáveis pela maior indicação de SNG e se nas maternidades onde estes bebês nasceram há fonoaudiólogo na equipe.

Acredita-se que uma forma de reduzir a indicação excessiva e desnecessária de SNG seja oferecer maiores informações sobre fissura labiopalatina nos cursos de graduação em Medicina, Fonoaudiologia e Enfermagem e capacitar profissionais das maternidades da região, para atuar com estes pacientes, conforme apontado anteriormente ${ }^{(2,4,18)}$. Com isso espera-se que tanto os bebês com fissura como seus pais possam ter experiências alimentares mais agradáveis e confortáveis.

\section{CONCLUSÃO}

A prevalência do uso de SNG em bebês portadores de fissura de lábio e/ou palato foi considerada alta, tendo em vista que estes bebês nasceram a termo e não apresentavam outros comprometimentos associados à fissura.

Não houve associação entre o uso de SNG e o tipo de fissura, bem como entre o uso de SNG e o tipo de maternidade. Houve associação entre o uso de SNG e a cidade de origem, sendo a indicação de sonda mais frequente em maternidades da região metropolitana de Belo Horizonte.

Metade dos bebês da amostra total estudada chegou ao centro especializado com até 33 dias de vida, sendo que o grupo que fez uso de SNG chegou mais precocemente do que o sem sonda. 


\begin{abstract}
Purpose: To investigate the prevalence of the use of nasogastric tube in babies with cleft lip and/or palate, and to correlate its use with type of cleft, maternity hospital (private or public) and city of origin, and age at the first visit to a cleft center. Methods: The sample consisted of data collected from 137 babies of both genders with cleft lip and/or palate, without any other associated anomalies, born full-term, who attended their first consultation at a specialized cleft center with ages between zero and 12 months (median=33 days). Statistical analyses used the coefficient of contingency test $(\mathrm{p}<0,05)$. Results: From the subjects, $61 \%$ were male and $39 \%$ were female; $51 \%$ presented cleft lip and palate, $35 \%$ cleft palate, and $14 \%$ cleft lip. Regarding place of birth, 36\% were born in private and $64 \%$ in public maternity hospitals; $60 \%$ were born in the city of Belo Horizonte (Minas Gerais, Brazil), $15 \%$ in other cities of the metropolitan area, and 25\% in other cities at the state of Minas Gerais (Brazil). The use of nasogastric tube was reported in 23\% of the cases. There was no association between the type of cleft or maternity and the use of the tube, but the later was more frequent within the metropolitan area $(\mathrm{p}=0,007)$. Conclusion: The prevalence of the use of nasogastric tube in babies with cleft lip and/or palate was considered high since they were born full-term and without any other associated anomalies that would indicate the need of the tube. The use of nasogastric tube was more frequent in babies born at the metropolitan area of Belo Horizonte, when compared to other cities in the state of Minas Gerais (Brazil).
\end{abstract}

Keywords: Cleft lip; Cleft palate; Infants, newborn; Feeding methods; Enteral nutrition

\section{REFERÊNCIAS}

1. Altmann EBC, Vaz ACN, Paula MBSF, Khoury RBF. Tratamento precoce. In: Altmann EBC. Fissuras labiopalatinas. 4a ed. Barueri: PróFono; 1997. p. 291-324.

2. Di Ninno CQMS, Gomes RO, Santo PG, Bueno MG, Galvão DA, Meira $\mathrm{AL}$, et al. O conhecimento de profissionais da área da saúde sobre fissura labiopalatina. Rev Soc Bras Fonoaudiol. 2004;9(2):93-101.

3. Paradise JL, Elster BA, Tan L. Evidence in infants with cleft palate that breast milk protects against otitis media. Pediatrics. 1994;94(6 Pt 1):853-60.

4. Amstalden-Mendes LG, Magna LA, Gil-da-Silva-Lopes VL. Neonatal care of infants with cleft lip and/or palate: feeding orientation and evolution of weight gain in a nonspecialized Brazilian hospital. Cleft Palate Craniofac J. 2007;44(3):329-34.

5. Pachi PR. Aspectos pediátricos. In: Altmann EBC. Fissuras labiopalatinas. 4a ed. Barueri: Pró-Fono; 1997. p. 283-8.

6. Di Ninno CQMS. Informações que os pais de bebês com fissura labiopalatina gostariam de receber no período neonatal. Rev Soc Bras Fonoaudiol. 2006;11(1):10-6.

7. Cavalheri VAN. Fissura labiopalatal e aleitamento materno. Rev CEFAC. 2000;2(1):53-65.

8. da Silva Dalben G, Costa B, Gomide MR, Teixeira das Neves LT. Breast-feeding and sugar intake in babies with cleft lip and palate. Cleft Palate Craniofac J. 2003;40(1):84-7.

9. Masarei AG, Sell D, Habel A, Mars M, Sommerlad BC, Wade A. The nature of feeding in infants with unrepaired cleft lip and/or palate compared with healthy noncleft infants. Cleft Palate Craniofac J. 2007;44(3):321-8

10. Silva EB, Fúria CLB, Di Ninno CQMS. Aleitamento materno em recém nascidos portadores de fissura labiopalatina: dificuldades e métodos utilizados. Rev CEFAC. 2005;7(1):21-8.

11. Oliver RG, Jones G. Neonatal feeding of infants born with cleft lip and/ or palate: parental perceptions of their experience in south Wales. Cleft Palate Craniofac J. 1997;34(6):526-32.

12. Reid J, Kilpatrick N, Reilly S. A prospective, longitudinal study of feeding skills in a cohort of babies with cleft conditions. Cleft Palate Craniofac J. 2006;43(6):702-9.

13. Reid J, Reilly S, Kilpatrick N. Sucking performance of babies with cleft conditions. Cleft Palate Craniofac J. 2007;44(3):312-20.

14. Montagnoli LC, Barbieri MA, Bettiol H, Marques IL, Souza L. Prejuízo no crescimento de crianças com diferentes tipos de fissura lábio-palatina nos 2 primeiros anos de idade: um estudo transversal. J. Pediatr (Rio J.). 2005; 81(6):461-5.

15. Trenouth MJ, Campbell AN. Questionnaire evaluation of feeding methods for cleft lip and palate neonates. Int J Paediatr Dent. 1996;6(4):241-4.

16. Redford-Badwal DA, Mabry K, Frassinelli JD. Impact of cleft lip and/or palate on nutritional health and oral-motor development. Dent Clin North Am. 2003;47(2):305-17.

17. Bachega MI, Thomé S, Capelozza Filho L. O uso de mamadeiras ortodônticas para a alimentação de crianças com fissuras lábio-palatais. Pediatr Mod. 1985;20(7):367-8, 371.

18. Smedegaard L, Marxen D, Moes J, Glassou EN, Scientsan C. Hospitalization, breast-milk feeding, and growth in infants with cleft palate and cleft lip and palate born in Denmark. Cleft Palate Craniofac J. 2008;45(6):628-32.

19. Waitzberg DL, Fadul RA, Aanholt DPJV, Plopper C, Terra RM. Indicações e Técnicas de Ministração em Nutrição Enteral. In: Waitzberg DL. Nutrição oral, enteral e parenteral na prática clínica. 3a ed. São Paulo: Atheneu; 2004. Vol.1. p. 561-71.

20. Guest J, Murray N, Antonson D. Continuous nasogastric feeding in paediatric patients. Nutr Support Serv. 1982;2:34-41.

21. Anderson GC, Vidyasagar D. Development of sucking in premature infants from 1 to 7 days post birth. Birth Defects Orig Artic Ser. 1979;15(7):145-71.

22. Teixeira Neto F. Nutrição enteral. In: Teixeira Neto F. Nutrição clínica. Rio de Janeiro: Guanabara Koogan; 2003. p. 234-42.

23. Field T, Ignatoff E, Stringer S, Brennam J, Greenberg R, Widmayer S, Anderson GC. Nonnutritive sucking during tube feedings: effects on preterm neonates in an intensive care unit. Pediatrics. 1982;70(3):381-4.

24. Marques IL, Sousa TV, Carneiro AF, Peres SPBA, Barbieri MA, Bettiol H. Sequência de Robin: protocolo único de tratamento. J Pediatr (Rio J). 2005;81(1):14-22.

25. Spina V, Psillakis JM, Lapa FS, Ferreira MC. Classificação das fissuras lábio-palatinas: sugestão de modificação. Rev Hosp Clin Fac Med São Paulo. 1972;27(1):5-6.

26. Capelozza Filho L, Silva Filho OG. Fissuras labiopalatinas. In: Petrelli E, coordenador. Ortodontia para fonoaudiologia. Curitiba: Lovise; 1994. p.197-239.

27. Modolin MLA, Cerqueira EMM. Etiopatogenia. In: Altmann EBC. Fissuras Labiopalatinas. 4a ed. Barueri: Pró-Fono; 1997. p. 25-30.

28. Di Ninno CQMS. Abordagem fonoaudiológica atual nas fissuras labiopalatinas. In: Britto ATBO. Livro de fonoaudiologia. São José dos Campos: Pulso Editorial; c2005. p. 325-38.

29. Silva EB, Rocha CMG, Lage RR. O bebê com fissura labiopalatina: intervenção interdisciplinar. In: Jesus MSV, Di Ninno CQMS. Fissura labiopalatina: fundamentos para a prática fonoaudiológica. São Paulo: Roca; c2009. p. 10-28.

30. Lima RC, Silva EB, Rocha CMGR e Ferreira RHR. Síndromes associadas à fissura labiopalatina: atuação fonoaudiológica. In: Jesus MSV, Di Ninno CQMS. Fissura labiopalatina: fundamentos para a prática fonoaudiológica. São Paulo: Roca; c2009. p. 29-47. 